\title{
The Future of Psychiatry: Psychohygiene, Analysis of Social Functioning and Overcoming Self-Destruction in Schizophrenia Spectrum Disorders
}

\author{
Boltivets SI ${ }^{1 *}$, Bashynskyi $00^{2}$ and Zahariichuk $\mathrm{OV}^{2}$ \\ ${ }^{1}$ State Institute of Family and Youth Policy, Ukraine \\ ${ }^{2}$ Shupyk National University of Healthcare of Ukraine, Ukraine
}

*Corresponding author: Sergii Ivanovich Boltivets, State Institute of Family and Youth Policy, Ukraine, Tel: +380 6835049 86; Email: boltivetssergij@i.ua

\author{
Opinion \\ Volume 6 Issue 1
}

Received Date: January 28, 2022

Published Date: February 08, 2022

DOI: $10.23880 / \mathrm{mhrij}-16000160$

\section{How is Human Resilience Measured?}

A person's resilience is primarily measured by his life expectancy. If life expectancy becomes a conscious goal, there is a need for reliable psycho-hygienic knowledge to achieve it. The effectiveness of any knowledge is expressed in the degree of its use by man, and therefore arises from the understanding of the knowledge obtained. The diversity of research throughout the development of scientific psychology reflects the division into trends, directions, specializations, experiments, reflecting parts of the integrity of the study of human mental life, which when combined do not reflect human integrity - it is always a particle object of study. That is why the role of psychohygiene in overcoming self-destruction in schizophrenia spectrum disorders has not yet acquired sufficient research justification. Probably, this natural general civilizational tendency to restore the lost in the scientific period of development of psychological knowledge of integrity led to the need to develop vitacultural methodology, i.e. methodology of human life culture for the sake of its stability.

\section{The Culture of Future Mental Life}

The methodology of the culture of life covers the previously distinguished individual mental phenomena events, experiments, directions, currents of psychological knowledge, the understanding of which is necessary for the full mental health of man. The system of means used by man and society as a whole to maintain their own mental health and, consequently, health as a whole, which does not exist without this essential property, is, in its entirety, mental hygiene. These remedies are contained in the mental health of man and in society itself and are unconsciously demanded in everyday life, just as the need for homeostasis is not realized until the time when it becomes clear that special efforts are needed to maintain the integrity of their own functioning. So, these are the means of preserving the mental state and the efforts made in order not to lose it. This is impossible without realizing the value of what is, because the unconscious in the self-preservation of every living being is certainly aimed at preserving its life as a whole.

Methodological bases of psychohygienic conditionality of development of psychological theories, their general civilization and individually significant for the person demand in a practical context of preservation of mental health of the person in communities where it's vital functioning takes place, open cyclic character of development of psychohygiene in interrelation with psychological societies. Risks and threats to their own integrity, life purpose and unimpeded realization of their natural capabilities. The psychological content of psychohygiene is the purpose of all branches of psychological knowledge to create a mental culture of man, including the culture of his own mental health. The current state of development of theoretical and methodological problems of psychohygiene indicates an irreversible process of restoring the humanistic orientation of public consciousness, and with it - the appeal of psychological research to the problems of social tact, attentiveness, prudence, respect for human dignity, care for mental and physical health. In the world psychological culture has a particularly significant tradition. This tradition is a reflection of the extremely tragic history of our civilization. Centuries 


\section{Mental Health \& Human Resilience International Journal}

of extermination of peoples on the lands of their own ethnic existence, and to this contributed by military means a large number of competing peoples, led to the development of reflexive abilities for self-preservation. Both genetically inherited and supported by family upbringing abilities of heightened sensitivity, compassion, humanity, which have been cultivated and continue to be cultivated from generation to generation for the sake of the ability to save and protect the health and life of everyone, resisted destruction.

\section{Problems that are Constantly Being Solved}

Suicidal and self-destructive behaviour is one of the most pressing problems in world psychiatry and psychotherapy. Auto aggressive actions under the influence of psychopathological experiences (delusions, hallucinations) of mentally ill people who are in a state of psychosis are manifestations of this aggression towards themselves. In patients with psychotic disorders of the schizophrenic spectrum during the acute state and in remission are insufficiently studied manifestations of exacerbation of psychopathological symptoms in certain periods of life, although in other periods of life they are virtually absent. Based on this, it is necessary to study the structural and dynamic characteristics of patients with psychotic disorders of the schizophrenic spectrum, taking into account different pathogenetic mechanisms. It is necessary to develop criteria for the differential diagnosis of manifestations of selfinjurious behavior in patients with psychotic disorders of the schizophrenic spectrum and to improve measures for their medical and social rehabilitation.

The main research attention should be focused on the spectrum of manifestations of suicide attempts, thoughts, intentions, self-harm within hallucinatory-delusional and affective-delusional syndromes in patients with psychotic disorders of the schizophrenic spectrum with different duration of the disease. Disorders of social functioning and quality of life of patients with schizophrenia are among the most relevant in modern psychiatry. Therefore, social dysfunction in patients with delusions in schizophrenia spectrum disorders should be properly studied. Increasing the level of their social rehabilitation involves improving the criteria for the differential diagnosis of delusions in schizophrenia spectrum disorders.

In the near future it is necessary:

- To determine the differential diagnostic criteria for delusions in patients with various disorders of the spectrum of schizophrenia.

- To find out the mechanisms of formation of delusional ideas of influence and their role in the violation of social functioning.

- Investigate the features of social dysfunction in patients with looming effects in disorders of the spectrum of schizophrenia.

- To find out the influence of the severity of these symptoms on the violation of social functioning.

- To develop an algorithm for providing rehabilitation measures for patients with looming effects in disorders of the spectrum of schizophrenia, taking into account the differential features of their formation.

\section{Conclusion}

The development of problems of psychohygiene, which is carried out mainly in the field of medicine, each time leads to the need for a comprehensive study of its psychological and, consequently, social dimensions. The idea of combining the practical efforts of teachers, physicians and psychologists for the institutional development of psycho-hygienic measures is becoming more and more pronounced in scientific research. Recent decades have strengthened the psychological, experimental focus of psychohygiene as a holistic field of knowledge and means of maintaining and strengthening mental health. The current period is characterized by increasing interest of researchers in psychohygienic issues, but most work on this issue is carried out in the field of medical sciences, which outlines the still little-studied field of social psychohygiene in overcoming self-destruction in schizophrenia. Some methodological approaches, methods of influence have already been accumulated by generalizing and systematizing psychological and hygienic experiments. Solving the problems of social psychohygiene requires integrity and defining sufficient and necessary boundaries of medical, social and psychological spheres of methodological, theoretical, methodological and practical solutions to the problems of maintaining and strengthening mental health.

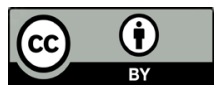

Boltivets SI, et al. The Future of Psychiatry: Psychohygiene and Overcoming Self-Destruction in Schizophrenia Spectrum Disorders. Ment Health Hum Resilience Int J 2022, 6(1): 000160. 